\title{
Interleukin-6 in Amniotic Fluid: A Reliable Marker for Adverse Outcomes in Women in Preterm Labor and Intact Membranes
}

\author{
Alfredo Leaños-Miranda ${ }^{a}$ Ana Graciela Nolasco-Leaños ${ }^{a}$ b \\ Reyes Ismael Carrillo-Juárez ${ }^{a} \quad$ Carlos José Molina-Pérez ${ }^{a} \quad$ Irma Isordia-Salasc \\ Karla Leticia Ramírez-Valenzuelad \\ aUnidad de Investigación Médica en Medicina Reproductiva, UMAE-Hospital de Ginecología y Obstetricia "Luis

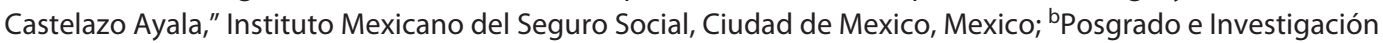 \\ Biomedicina y Biotecnología Molecular, Instituto Politécnico Nacional, Ciudad de Mexico, Mexico; 'Unidad de \\ Investigación en Trombosis, Hemostasia y Aterogénesis, HGR No.1 "Dr. Carlos Mac Gregor," Instituto Mexicano del \\ Seguro Social, Ciudad de Mexico, Mexico; 'Division de Medicina Materno-Fetal, UMAE-Hospital de Ginecología y \\ Obstetricia "Luis Castelazo Ayala," Instituto Mexicano del Seguro Social, Ciudad de Mexico, Mexico
}

\section{Keywords}

Preterm labor · Interleukin-6 · Amniotic fluid ·

Intra-amniotic inflammation · Adverse outcomes

\begin{abstract}
Introduction: Amniotic fluid (AF) interleukin-6 (IL-6) concentration has been associated to preterm delivery and perinatal morbidity and mortality in women with preterm labor and intact membranes. Nevertheless, the clinical significance of this biomarker of intra-amniotic inflammation (IAI) is still unclear due in part to the paucity of large studies. Methods: AF IL- 6 concentrations were determined in 452 consecutive women with preterm labor and intact membranes, categorized into 3 groups: 302 without IAI (IL-6 of $<2.6 \mathrm{ng} / \mathrm{mL}$ ), 64 with mild IAI (IL-6 of $2.6-11.2 \mathrm{ng} / \mathrm{mL}$ ), and 86 with severe IAI (IL- 6 of $\geq 11.3 \mathrm{ng} / \mathrm{mL}$ ). Results: The severe IAI group had a short pregnancy duration from amniocentesis to delivery (median 3 days) than in without IAI group (median 45 days); meanwhile, the mild IAI group had a latency that was intermediate to the severe and without IAI groups
\end{abstract}

(median 9.5 days). As compared to women without IAI, women with mild and severe IAI had higher rates of preterm delivery at both $<34$ and $<37$ weeks of gestation and perinatal morbidity and mortality. Furthermore, the risk of various individual adverse outcomes (short latency from amniocentesis to delivery [at $\leq 3$ days, $\leq 7$ days, and $\leq 14$ days], preterm delivery at both $<34$ and $<37$ weeks of gestation, histologic chorioamnionitis, respiratory distress syndrome, and congenital sepsis) was higher in women with severe IAI (OR $\geq$ 2.8), compared with women without IAI. Conclusions: AF IL-6 concentrations appear to be suitable marker to assess the degree of IAI and are associated with increased risk of adverse outcomes.

(C) 2021 S. Karger AG, Basel

\section{Introduction}

Preterm delivery affects $9.63 \%$ of all pregnancies in the USA, with a similar prevalence (9.14\%) among Hispanic pregnancies [1]. Preterm labor with intact membranes 
account for about one-third of preterm births, leading to significant perinatal mortality and morbidity, which are closely associated to the degree of prematurity and intrauterine infection [2-4]. Although microbial invasion of the amniotic cavity in patients with preterm labor and intact membranes is a main risk for the progression of labor to preterm delivery, however, in women with preterm labor with intact membranes, the rate of positive amniotic fluid (AF) cultures ranges from 9.2 to $18 \%$ [47]. This may partly be due to the limitations of standard culturing methods employed in hospital laboratories, which are unable to detect the uncultivated species, even by using PCR amplification; the rate to detect bacteria in AF ranges from 8.5 to $55 \%[4,7,8]$.

On the other hand, between 30.6 and $36.1 \%$ of women in preterm labor and intact membranes have demonstrable intra-amniotic inflammation (IAI), defined as elevated proinflammatory cytokine levels in AF such as interleukin-6 (IL-6) $[4,9]$, even with a negative AF culture $(21.4-27.2 \%)(4,9)$; likewise, to intra-amniotic infection, IAI is strongly associated with short latency and high rates of perinatal morbidity and mortality $[4-6,9]$ and may also contribute to the pathogenesis of adverse shortand long-term neonatal pulmonary and neurologic sequelae $[3,10,11]$.

Since in our hospital, the rate of positive AF cultures is practically nil, a more accurate prediction of preterm delivery is clinically important for improving pregnancy management and for counseling women with preterm labor. On the other hand, our renewed interest of the test that detects the presence of IAI comes from a recent study showing that in patients with preterm labor and intact membranes with intra-amniotic infection or IAI (mostly with an AF culture negative for microorganisms), the eradication of IAI or intra-amniotic infection after treatment with antibiotics was confirmed in $79 \%$ of patients [12]. The aims of this study were to compare the outcomes of preterm labor in women with and without IAI and to examine whether the outcomes are related to the severity of inflammation according to IL-6 levels in AF.

\section{Material and Methods}

We carried out a prospective-observational cohort study. All women were consecutive patients admitted to our tertiary care level hospital (Hospital de Ginecología y Obstetricia "Luis Castelazo Ayala," IMSS. México City) between January 2016 and July 2019 with the diagnosis of spontaneous preterm labor and intact membranes ( $<36$ weeks of gestation) and who underwent amniocentesis. The exclusion criteria were uterine anomalies, multifetal preg- nancy, preterm premature rupture of membranes, prior or subsequent cerclage, major fetal anomaly, fetal aneuploidy, or a medical indication for preterm birth. Preterm labor was defined as regular uterine contractions, and cervical change (softening, effacement, cervical length by transvaginal sonography $\leq 30 \mathrm{~mm}$, or dilatation) that required hospitalization. Gestational age was determined based on the last menstrual period and the first trimester obstetrical ultrasound evaluation, when available.

AF was obtained by transabdominal amniocentesis under sonographic guidance and aseptic conditions. The AF was immediately sent to local hospital laboratory for culture, assessment of glucose concentration and white blood cell count. Ten $\mathrm{mL}$ of AF was centrifuged at $3,000 \mathrm{rpm}$ and $4^{\circ} \mathrm{C}$ for $15 \mathrm{~min}$, aliquoted, and stored at $-80^{\circ} \mathrm{C}$ until assayed.

Clinical chorioamnionitis was defined as antenatal maternal fever $\left(\geq 37.8^{\circ} \mathrm{C}\right)$ plus 2 or more of the following criteria: maternal leukocytosis $\left(>15,000\right.$ cells $\left./ \mathrm{mm}^{3}\right)$, uterine tenderness, malodorous vaginal discharge, or fetal tachycardia ( $>160$ beats $/ \mathrm{min}$ ) [13]. Histologic chorioamnionitis was defined in the presence of acute inflammatory changes on examination of a membrane roll and chorionic plate of the placenta [14]. The adverse neonatal outcomes studied included preterm delivery, the death (stillbirths [defined as death of a fetus] and neonatal death [defined as death a newborn until hospital discharge]), neonatal sepsis, and respiratory distress syndrome.

Management of preterm labor, the decision to use antibiotics, and other clinical decisions were left to the discretion of the attending obstetrician. Clinicians had access to local hospital laboratory results but were blinded to the AF IL- 6 results. The IL- 6 concentrations in the stored $\mathrm{AF}$ were measured in duplicate using a quantitative sandwich enzyme immunoassay (Quantikine ELISA \#D6050; R\&D Systems, Minneapolis, MN, USA) following the manufacturer's instructions. The intra- and inter-assay coefficients of variations were 5.7 and $6.2 \%$, respectively.

Patients were classified into 3 groups according to the results of AF concentrations of IL-6, using the cutoff points that have been determined by previous studies $[4,5,9]$ : (1) no IAI, IL- 6 concentration of $<2.6 \mathrm{ng} / \mathrm{mL}$; (2) mild IAI, IL- 6 concentration of 2.6 to $<11.3 \mathrm{ng} / \mathrm{mL}$; and (3) severe IAI, IL- 6 concentration of $\geq 11.3 \mathrm{ng} /$ $\mathrm{mL}$.

\section{Statistical Analysis}

Differences between categorical variables were determined by the $\chi^{2}$ test with Yates' continuity correction or the Fisher's exact test for small samples (or the Mantel-Haenszel $\chi^{2}$ test with linear tendency for variables with more than 2 categories). Differences among $\geq 3$ continuous variables were determined by one-way ANOVA followed by post hoc procedures (Scheffe's test) or by the Kruskal-Wallis one-way test followed by the Mann-Whitney U test for nonnormally distributed variables. Time to delivery according to IAI assessed by AF IL- 6 concentrations was evaluated using Kaplan-Meier curves and Cox proportional hazard models. Logistic regression analysis was used to adjust the odds ratios for gestational age at delivery, cervical dilatation $\geq 2 \mathrm{~cm}$, cervical length, presence of sludge, and AF glucose levels since these variables were significantly different among the groups studied by univariate analysis. Moreover, inclusion of these variables in the models were necessary considering that these variables may distinctly affect the latency and the adverse outcomes. A two-tailed $p<0.05$ was considered statistically significant. 


\section{Results}

\section{General Description of the Population Studied}

A total of 452 consecutive women with the diagnosis of preterm labor and intact membranes were included in the study. There were no complications attributed to amniocentesis. IAI was found in 150 women (33.2\%), which included 64 cases with mild IAI and 86 cases with severe IAI. Both mild and severe IAI were more frequent at earlier gestational ages (Fig. 1), mainly at $<24$ weeks ( $p \leq$ 0.02 , compared with the rest of the groups by gestational age at amniocentesis). The clinical characteristics of the patients are shown in Table 1. There were no significant differences among study groups in terms of maternal age, BMI, gravidity, nulliparity, prior miscarriage, history of preterm birth, use of tocolytics, antenatal corticosteroids administration, or use of antibiotics. Women with IAI (both mild and severe) were more likely to have lower cervical length measurements and had a greater proportion of cervical dilation $\geq 2 \mathrm{~cm}$ compared with women without IAI. Compared with no IAI and mild IAI women, patients with severe IAI had lower gestational age at amniocentesis, a greater proportion of sludge, as well as lower AF glucose levels. As expected, the frequency of positive AF culture was practically nil (0.7\% [3/452]), including one isolate each of Acinetobacter spp, Enterococcus spp, and Candida albicans.

\section{Relationship between IAI and Pregnancy Duration} from Amniocentesis to Delivery

Table 2 shows the interval from amniocentesis to delivery. The severe IAI group had significantly shorter latency (median 3 days) than that of the groups with mild IAI and no IAI (median 9.5 and 45 days, respectively). In addition, the latency was significantly shorter in mild IAI group than in no IAI group. Kaplan-Meier curves depicting the probability of remaining undelivered for women stratified by severity of IAI are shown in Figure 2. Both mild and severe IAI were associated with shorter time to delivery, as compared to those with no IAI $(p<0.001)$. Cox proportional hazard models after adjustment for gestational age at amniocentesis, cervical dilation $\geq 2 \mathrm{~cm}$, cervical length, sludge, and AF glucose levels showed that mild and severe IAI were associated with increased risk of early delivery (HR: 2.4, 95\% CI 1.8-3.2 and HR: 31.8, $95 \%$ CI 21.2-47.7, respectively) compared to no IAI.

\section{Relationship between IAI with Adverse Outcomes}

Comparison of pregnancy and perinatal outcomes of studied patients is shown in Table 2. Compared with no

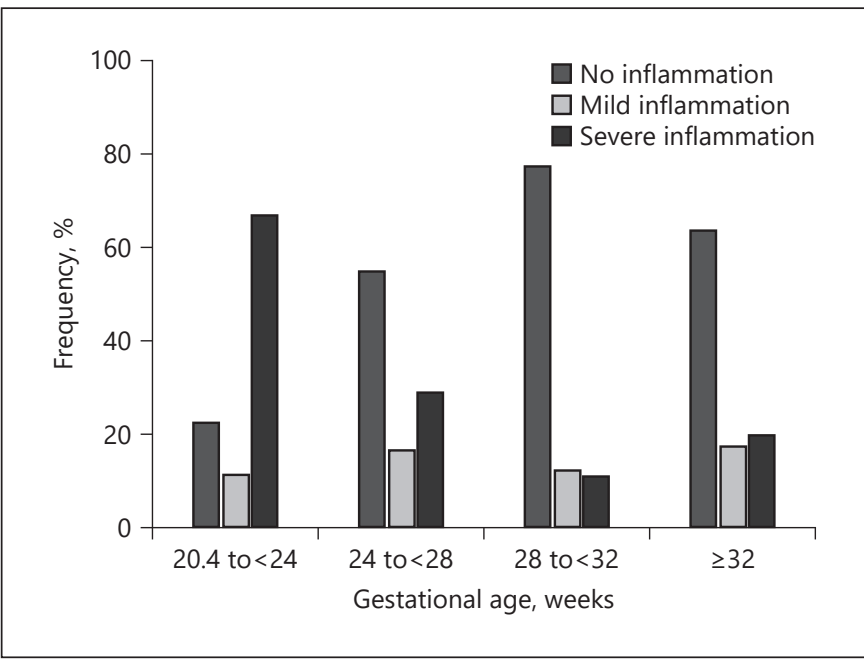

Fig. 1. Frequency of IAI (according to AF IL-6 concentrations) as a function of gestational age at amniocentesis. Both mild and severe intra-amniotic inflammation were more prevalent at $<24$ weeks of gestational age, compared with the rest of the groups by gestational age $(p \leq 0.02)$. AF, amniotic fluid; IAI, intra-amniotic inflammation.

IAI women, patients with mild and severe IAI had lower gestational age at delivery, delivered infants with lower birth weights and lower Apgar scores, and had a greater proportion of preterm delivery (both at $<34$ and $<37$ weeks of gestation), respiratory distress syndrome, congenital sepsis, and perinatal deaths. In addition, all these adverse outcomes were more pronounced in women with severe IAI than in those with mild IAI. Women with severe IAI had the highest proportion of histologic chorioamnionitis, whereas the proportions of histologic chorioamnionitis were not significantly different between women with mild IAI and no IAI. Clinical chorioamnionitis were uncommon in all 3 groups.

Logistical regression analyses were used to determine the association between severity of IAI and risk of adverse outcomes (Table 3). The risk of shorter latency (at $\leq 3, \leq 7$, and $\leq 14$ days) and of preterm delivery (both at $<34$ and $<37$ weeks of gestation) was progressively higher as severity of IAI increased, suggesting that the relationship between severity of IAI and risk of these outcomes has a linear dose-response pattern. Women with severe IAI only exhibited higher risk for histologic chorioamnionitis, respiratory distress syndrome, and congenital sepsis. With regard to the risk of perinatal deaths, there were no significant differences according to severity of IAI. 
Table 1. Clinical characteristics of patients with preterm labor and intact membranes according to IAI assessed by AF IL-6 concentrations

\begin{tabular}{|c|c|c|c|c|}
\hline Variable & $\begin{array}{l}\text { No inflammation } \\
(n=302)\end{array}$ & $\begin{array}{l}\text { Mild inflammation } \\
(n=64)\end{array}$ & $\begin{array}{l}\text { Severe inflammation } \\
(n=86)\end{array}$ & $p$ value \\
\hline Maternal age, mean $\pm S D$, years & $29.2 \pm 6.2$ & $29.1 \pm 5.8$ & $29.8 \pm 5.6$ & \\
\hline $\mathrm{BMI}$, mean $\pm \mathrm{SD}$ & $28.1 \pm 4.5$ & $28.3 \pm 5.8$ & $28.8 \pm 4.1$ & \\
\hline Gravity, median (IQR) & $2(1-3)$ & $2(1-3)$ & $2(1-3)$ & \\
\hline Primigravid, $n(\%)$ & $122(40.4)$ & $20(31.3)$ & $24(27.9)$ & \\
\hline Miscarriage, median (IQR) & $0(0-1)$ & $0(0-1)$ & $0(0-1)$ & \\
\hline Prior preterm birth, $n(\%)$ & $56(18.5)$ & $16(25.0)$ & $16(18.6)$ & \\
\hline Cervical length, mean $\pm \mathrm{SD}, \mathrm{mm}$ & $13.0 \pm 6.2$ & $10.0 \pm 6.4^{*}$ & $9.7 \pm 7.5^{*}$ & $<0.001$ \\
\hline Cervical dilation $\geq 2 \mathrm{~cm},(\%)$ & $26(8.6)$ & $12(18.8)^{*}$ & $22(25.6)^{*}$ & $<0.001$ \\
\hline Sludge, $n(\%)$ & $28(9.3)$ & $4(6.3)$ & $30(34.9)^{*, \dagger}$ & $<0.001$ \\
\hline Gestational age at amniocentesis, mean $\pm \mathrm{SD}$, weeks & $29.6 \pm 2.5$ & $29.4 \pm 3.1$ & $27.7 \pm 3.6^{*, \dagger}$ & $<0.001$ \\
\hline Culture positive, $n(\%)$ & $1(0.3)$ & $1(1.6)$ & $1(1.2)$ & \\
\hline Glucose, mean $\pm \mathrm{SD}, \mathrm{mg} / \mathrm{dL}$ & $39.2 \pm 15.9$ & $32.7 \pm 12.2^{*}$ & $19.9 \pm 13.6^{*, \dagger}$ & $<0.001$ \\
\hline AF IL-6, median (IQR), ng/mL & $0.6(0.3-1.0)$ & $4.2(3.1-6.0)^{*}$ & $38.7(17.0-79.7)^{*, \dagger}$ & $<0.001$ \\
\hline Use of tocolytics, $n(\%)$ & $268(88.7)$ & $54(84.4)$ & $80(93.0)$ & \\
\hline Antenatal corticosteroid administration, $n(\%)$ & $277(91.7)$ & $56(87.5)$ & $81(94.2)$ & \\
\hline Use of antibiotics, $n(\%)$ & $17(5.6)$ & $5(7.8)$ & $10(11.6)$ & \\
\hline
\end{tabular}

IQR, interquartile range; AF, amniotic fluid; IL-6, interleukin-6; IAI, intra-amniotic inflammation. * $p$ value is given only for significant differences; versus no inflammation. ${ }^{\dagger} p$ value is given only for significant differences; versus mild inflammation, after application of appropriate statistical tests.

Table 2. Pregnancy and perinatal outcomes of patients with preterm labor and intact membranes according to IAI assessed by AF IL-6 concentrations

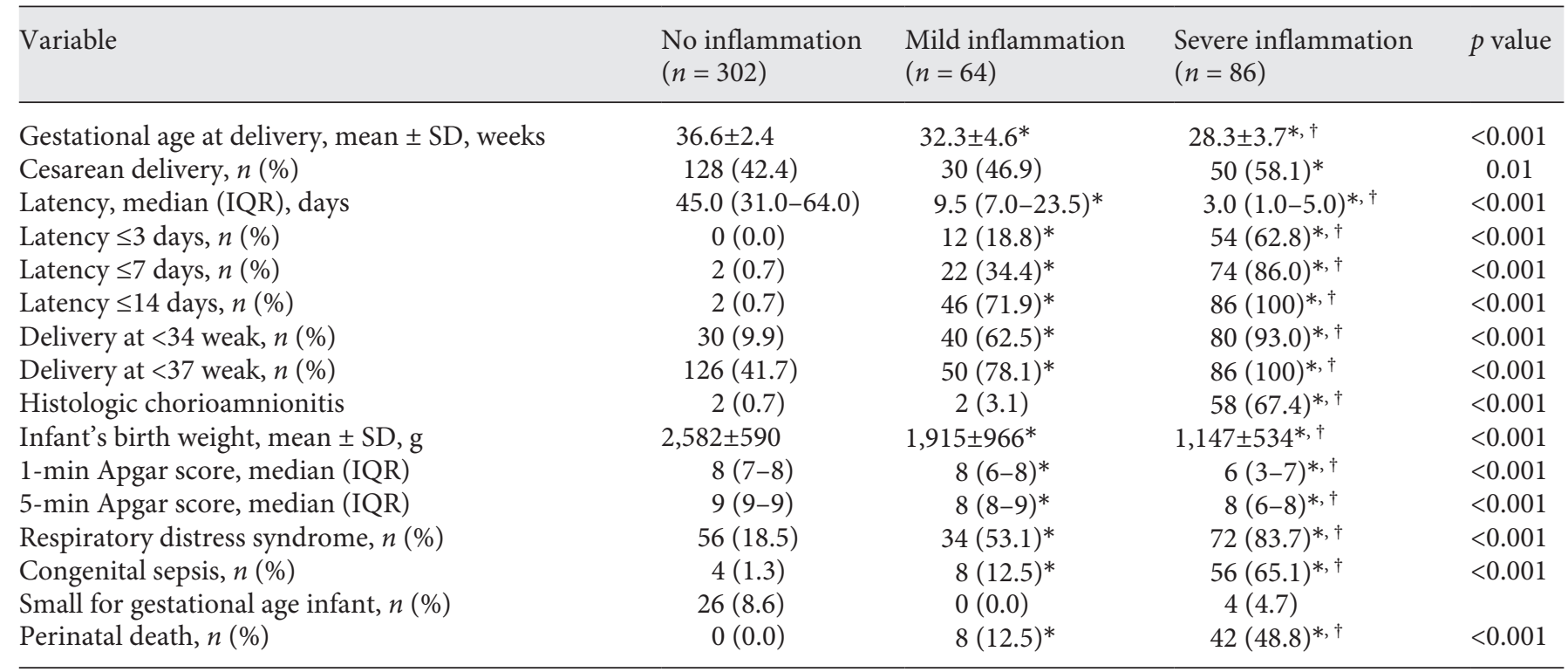

IQR, interquartile range; AF, amniotic fluid; IL-6, interleukin-6; IAI, intra-amniotic inflammation. * $p$ value is given only for significant differences; versus no inflammation. ${ }^{\dagger} p$ value is given only for significant differences; versus mild inflammation, after application of appropriate statistical tests. 
Fig. 2. Kaplan-Meier curves of pregnancy duration from amniocentesis to delivery in women with preterm labor and intact membranes according to the degree of IAI $(p<0.001$ for all comparison). IAI, intraamniotic inflammation.

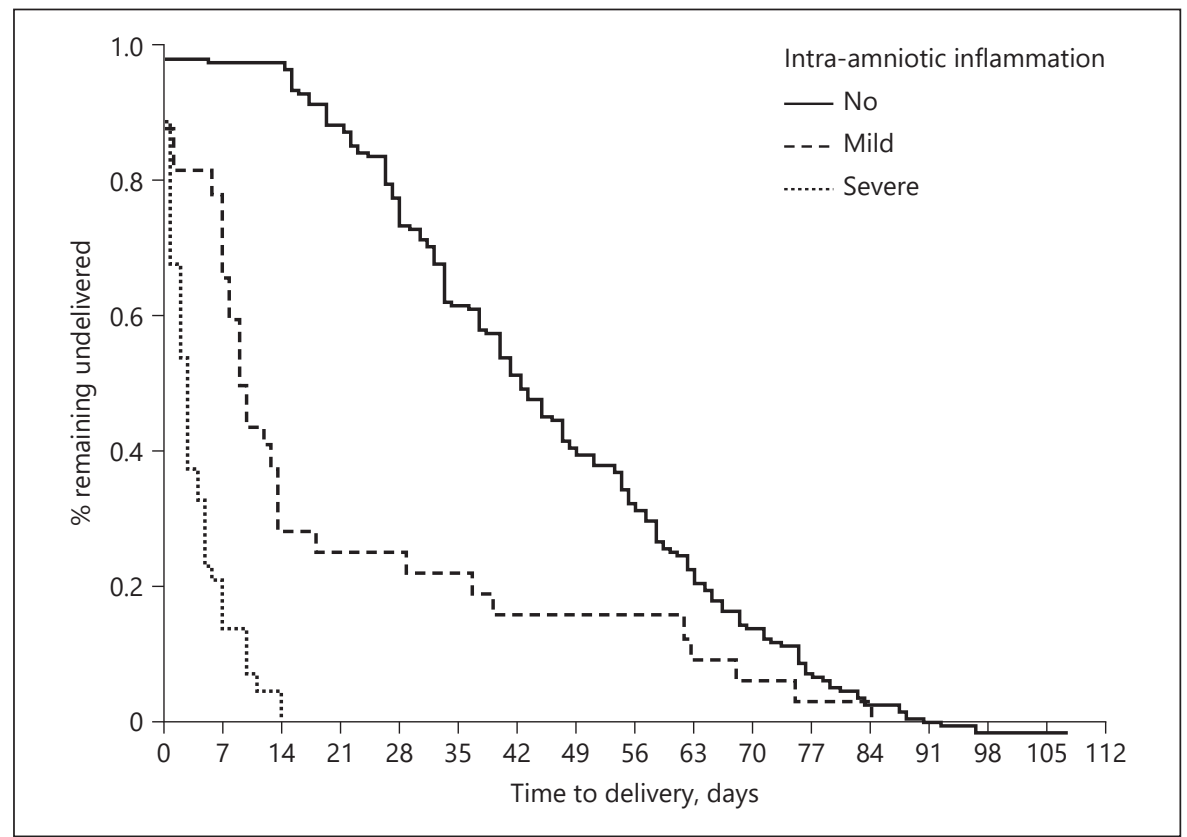

\section{Discussion}

In the present study, involving a large number of women in preterm labor with intact membranes, we found that a third of these women had an intra-amniotic inflammatory response as evidenced by elevation of IL-6, mainly at early gestational ages, as previously demonstrated in other studies $[4,9,15]$. Furthermore, we are able to find that both mild and severe IAI assessed by high AF IL-6 concentrations are associated with short latency and the subsequent development of high rates of perinatal morbidity and mortality. Moreover, severe IAI, which is defined by an AF IL- 6 concentration $\geq 11.3 \mathrm{ng} / \mathrm{mL}$, was associated with short latencies and high rates of perinatal morbidity and mortality compare to those with mild IAI [4].

Although previous studies have demonstrated that an AF IL- 6 concentration $\geq 11.3 \mathrm{ng} / \mathrm{mL}$ had a sensitivity of 87 $100 \%$ and specificity of $83-92 \%$ for the detection of microbial invasion of amniotic cavity $[4,5,16]$, however, a negative culture does not exclude intra-amniotic infection because many bacteria are resistant to culture with standard microbiologic techniques used in our clinical laboratory [8, 17]. In addition, our hospital does not have implemented techniques for the laboratory culture of Mycoplasma hominis or Ureaplasma urealyticum, which are well-known human pathogens responsible for intra-amniotic infection in preterm birth. The abovementioned can explain the rate nil of positive cultures in our study. Of interest is the finding that all women with an IL- $6 \geq 11.3 \mathrm{ng} / \mathrm{mL}$ delivered prema-

IL-6 in Amniotic Fluid as Marker of Intra-Amniotic Inflammation turely and had a high rate of histologic chorioamnionitis. Therefore, independent to the culture result, a high AF IL-6 concentration identified a subset of women at risk of impeding preterm delivery. We thus have confirmed and extended previous observations as to the relationship between the degree of IAI with clinical outcomes of women in preterm labor with intact membranes, independently of a demonstrable intra-amniotic infection $[4,5,16]$.

On the other hand, it has been suggested that mild IAI probably represents an early stage in a process that progresses to severe IAI over a period of days to weeks [4]. In this vein, in a nonhuman primate model of preterm labor induced by intra-amniotic infection, it has been demonstrated that the levels of cytokines, including IL-6, rise progressively over time after bacterial inoculation and correlate with the onset of uterine activity, and also of interest was the finding that clinical signs of infection occurred after the onset of uterine contractions [18]. In line with this idea, in women with mild IAI we found that AF IL-6 concentrations showed a significant negative correlation with pregnancy duration from amniocentesis to delivery $\left(r_{\mathrm{s}}=-0.65, p<0.001\right)$. However, our data do not support the possibility that IL-6 rise progressively over time because we did not perform serial amniocentesis, and further research is required to address this question.

Previous studies have demonstrated that women in preterm labor with intact membranes and high AF IL-6 concentrations are at higher risk for developing adverse outcomes independently of the presence of microbes $[4,5,9$, 
Table 3. Risk of adverse pregnancy outcomes according to IAI assessed by AF IL- 6 concentrations in patients with preterm labor and intact membranes

\begin{tabular}{|c|c|c|c|}
\hline Adverse outcome & $\begin{array}{l}\text { No inflammation }(n=302) \text {, } \\
\text { events OR, } n(95 \% \mathrm{CI}) \\
\text { single variable } \\
\text { multivariable }\end{array}$ & $\begin{array}{l}\text { Mild inflammation }(n=64) \text {, } \\
\text { events OR, } n(95 \% \mathrm{CI}) \\
\text { single variable } \\
\text { multivariable }\end{array}$ & $\begin{array}{l}\text { Severe inflammation }(n=86) \text {, } \\
\text { events OR, } n(95 \% \mathrm{CI}) \\
\text { single variable } \\
\text { multivariable }\end{array}$ \\
\hline Latency $\leq 3$ days & $\begin{array}{l}0 \\
\text { Reference* }\end{array}$ & 12 & $\begin{array}{l}54 \\
49.83(24.21-102.52) \\
\mathbf{4 5 . 9 3}(\mathbf{2 1 . 2 4 - 9 9 . 9 2 )}\end{array}$ \\
\hline Latency $\leq 14$ days & $\begin{array}{l}2 \\
\text { Reference }\end{array}$ & $\begin{array}{l}46 \\
383.33(86.12-1,706.83) \\
257.04(72.61-907.73)\end{array}$ & $\begin{array}{c}86 \\
20,791.61(988.91-337,231.2) \\
\mathbf{1 8 , 5 0 1 . 1 1}(\mathbf{9 8 1 . 4}-\mathbf{4 2 0 , 1 8 5 . 3 )}\end{array}$ \\
\hline Delivery at $<37$ weeks & $\begin{array}{l}126 \\
\text { Reference }\end{array}$ & $\begin{array}{l}50 \\
5.04(2.61-9.42) \\
\mathbf{5 . 1 1}(\mathbf{2 . 7 1 - 9 . 7 2 )}\end{array}$ & $\begin{array}{r}86 \\
241.40(14.81-3,926.82) \\
\mathbf{5 2 . 1 0}(\mathbf{1 2 . 5 1 - 2 1 6 . 4 2 )}\end{array}$ \\
\hline Histologic chorioamnionitis & $\begin{array}{l}2 \\
\text { Reference }\end{array}$ & $\begin{array}{l}2 \\
4.80(0.71-35.04) \\
\mathbf{4 . 6 4}(\mathbf{0 . 6 1 - 3 3 . 5 3 )}\end{array}$ & $\begin{array}{l}58 \\
310.72(72.3-1,340.40) \\
\mathbf{2 6 6 . 7 0}(\mathbf{6 1 . 5 2 - 1 , 1 5 6 . 9 7 )}\end{array}$ \\
\hline Respiratory distress syndrome & $\begin{array}{l}56 \\
\text { Reference }\end{array}$ & $\begin{array}{l}34 \\
5.06(2.81-8.85) \\
\mathbf{1 . 6 3}(\mathbf{0 . 8 7 - 3 . 2 8 )}\end{array}$ & $\begin{array}{l}72 \\
22.66(11.91-42.93) \\
\mathbf{2 . 8 2}(\mathbf{1 . 3 3}-\mathbf{6 . 4 7 )}\end{array}$ \\
\hline Congenital sepsis & $\begin{array}{l}4 \\
\text { Reference }\end{array}$ & $\begin{array}{c}8 \\
10.65(3.11-36.54) \\
\mathbf{3 . 2 1}(\mathbf{0 . 8 8}-\mathbf{1 2 . 9 7 )}\end{array}$ & $\begin{array}{l}56 \\
139.17(47.11-410.28) \\
26.62(7.53-94.59)\end{array}$ \\
\hline
\end{tabular}

ORs were adjusted for gestational age at delivery, cervical dilation $\geq 2 \mathrm{~cm}$, cervical length, presence of sludge, and amniotic fluid glucose levels. The reference* OR is the frequency of adverse outcomes in no intra-amniotic inflammation and mild intra-amniotic inflammation combined. Non-bold indicates the significance for univariate analysis (single variable). Bold indicates the significance for multivariate analysis. OR, odds ratio; CI, confidence interval; AF, amniotic fluid; IL-6, interleukin-6; IAI, intra-amniotic inflammation.

19]. We also assessed the association between the degree of IAI serum and the individual risks of adverse pregnancy outcomes. Women with severe IAI showed an increased risk of all specific adverse outcomes studied. Furthermore, the risk of various individual adverse outcomes, including short latency from amniocentesis to delivery (at $\leq 3, \leq 7$, and $\leq 14$ days), preterm delivery at both $<34$ and $<37$ weeks of gestation, histologic chorioamnionitis, respiratory distress syndrome, and congenital sepsis, was progressively higher as the degree of IAI increased, even after taking into account gestational age at delivery, cervical dilatation $\geq 2$ $\mathrm{cm}$, cervical length, presence of sludge, and AF glucose levels. We thus have confirmed and extended previous observations [4] as to the association between the degree of IAI at the time of initial evaluation and subsequent adverse outcomes in women in preterm labor with intact membranes. However, and in contrast to the data presented herein, in that study were unable to find an increased risk for the development of combined or any individual adverse outcomes in women with mild or severe IAI. This 
discrepancy may stem from differences in the population studied and/or the limited sample size of alternative adverse outcomes studied, leading to a low power of study results. Although on univariate analysis, we found that mild and severe IAI were associated with the risk of perinatal deaths. When adjusting for gestational age at delivery on logistic regression analysis, there were no significant differences, suggesting that the degree of prematurity is the major determinant of neonatal mortality.

The identification of women in preterm labor and intact membranes with IAI (assessed by AF IL- 6 concentrations) and who are at increased risk for early preterm delivery and adverse outcomes would have substantial clinical implications. In this regard, it has been recently shown that the administration of antibiotics to women with preterm labor and intact membranes can eradicate intra-amniotic infection or IAI in a subset of these women [12].

The strengths of our study include a well-defined, large population of women in preterm labor and intact membranes who had received the same standardized medical protocol, and the fact that all caregivers were blinded to IL-6 results, which evidently avoided a selection bias. To rule out the effects of potential confounders, the estimated odds ratios were adjusted for other variables significant in univariate analysis. On the other hand, we are aware of the presence of certain limitations, including the high frequency of negative cultures, precluding the analysis of other outcomes, such as intra-amniotic infection; however, the frequency of adverse outcomes is in line with a previous multicenter study which demonstrated that adverse outcomes are related more closely to IAI than to the presence of microbes [4].

\section{Conclusion}

Our results demonstrate that IAI complicates onethird of women with preterm labor and intact membranes, especially at early gestational ages. The degree of
IAI, assessed by AL IL- 6 concentrations is closely associated with the risk of adverse outcomes in women with preterm labor and intact membranes. Measurement of AF IL-6 has the potential relevance as a biomarker for risk stratification of adverse outcomes in these women. Further prospective longitudinal studies are still needed to assess the potential role of AF IL- 6 in the diagnostic and management of women in preterm labor and intact membranes.

\section{Statement of Ethics}

The study protocol was approved by the Scientific and Ethics Committees of Instituto Mexicano del Seguro Social (R-20153606-30. Date of approval 2015-07-14) and conforms to the ethical guidelines of the 1975 Declaration of Helsinki modified in 2013. All participating subjects signed an informed consent before enrollment.

\section{Conflict of Interest Statement}

The authors have no conflicts to declare.

\section{Funding Sources}

This study was supported by El Fondo de Investigación en Salud grant (FIS/IMSS/PROT/G16/1585), Mexico to A.L.-M.

\section{Author Contributions}

Alfredo Leaños-Miranda and Karla Leticia Ramírez-Valenzuela contributed equally to this study. A.L.-M. and K.L.R-V. designed the study, analyzed and interpreted the data, and wrote the final version of article. The remaining co-authors performed the ELISA, recruited patients, collected biological specimens, and followed the patients prospectively to the point of delivery. All co-authors participated in interpretation of the data and approved the final version.

\section{References}

1 Martin JA, Hamilton BE, Osterman MJ. Births in the United States, 2015. NCHS Data Brief. $2016 \operatorname{Sep}(258): 1-8$.

2 Bulletins-Obstetrics ACoP. ACOG practice bulletin no. 80: premature rupture of membranes. Clinical management guidelines for obstetrician-gynecologists. Obstet Gynecol. 2007 Apr;109(4):1007-19.

3 Jung EY, Park KH, Han BR, Cho SH, Yoo HN, Lee J. Amniotic fluid infection, cytokine levels, and mortality and adverse pulmonary, in- testinal, and neurologic outcomes in infants at 32 weeks' gestation or less. J Korean Med Sci. 2017 Mar;32(3):480-7.

4 Combs CA, Gravett M, Garite TJ, Hickok DE, Lapidus J, Porreco R, et al. Amniotic fluid infection, inflammation, and colonization in preterm labor with intact membranes. Am J Obstet Gynecol. 2014 Feb;210(2):125.e1e15.

5 Romero R, Yoon BH, Mazor M, Gomez R, Diamond MP, Kenney JS, et al. The diagnostic and prognostic value of amniotic fluid white blood cell count, glucose, interleukin-6, and gram stain in patients with preterm labor and intact membranes. Am J Obstet Gynecol. 1993 Oct;169(4):805-16.

6 Greig PC, Ernest JM, Teot L, Erikson M, Talley R. Amniotic fluid interleukin-6 levels correlate with histologic chorioamnionitis and amniotic fluid cultures in patients in premature labor with intact membranes. Am J Obstet Gynecol. 1993 Oct;169(4):1035-44.
IL-6 in Amniotic Fluid as Marker of Intra-Amniotic Inflammation
Fetal Diagn Ther 2021;48:313-320 DOI: $10.1159 / 000514898$ 
7 Markenson GR, Martin RK, Tillotson-Criss M, Foley KS, Stewart RS Jr, Yancey M. The use of the polymerase chain reaction to detect bacteria in amniotic fluid in pregnancies complicated by preterm labor. Am J Obstet Gynecol. 1997 Dec;177(6):1471-7.

8 DiGiulio DB, Romero R, Amogan HP, Kusanovic JP, Bik EM, Gotsch F, et al. Microbial prevalence, diversity and abundance in amniotic fluid during preterm labor: a molecular and culture-based investigation. PLoS One. 2008 Aug 26;3(8):e3056.

9 Yoon BH, Romero R, Moon JB, Shim SS, Kim $\mathrm{M}$, Kim G, et al. Clinical significance of intraamniotic inflammation in patients with preterm labor and intact membranes. Am J Obstet Gynecol. 2001 Nov;185(5):1130-6.

10 Yoon BH, Jun JK, Romero R, Park KH, Gomez R, Choi JH, et al. Amniotic fluid inflammatory cytokines (interleukin-6, interleukin1beta, and tumor necrosis factor-alpha), neonatal brain white matter lesions, and cerebral palsy. Am J Obstet Gynecol. 1997 Jul;177(1): 19-26.
11 Yoon BH, Romero R, Park JS, Kim CJ, Kim $\mathrm{SH}$, Choi JH, et al. Fetal exposure to an intraamniotic inflammation and the development of cerebral palsy at the age of three years. Am J Obstet Gynecol. 2000 Mar; 182(3):675-81.

12 Yoon BH, Romero R, Park JY, Oh KJ, Lee J, Conde-Agudelo A, et al. Antibiotic administration can eradicate intra-amniotic infection or intra-amniotic inflammation in a subset of patients with preterm labor and intact membranes. Am J Obstet Gynecol. 2019 Aug; 221(2):142-e22.

13 Gibbs RS, Blanco JD, St Clair PJ, Castaneda YS. Quantitative bacteriology of amniotic fluid from women with clinical intraamniotic infection at term. J Infect Dis. 1982 Jan;145(1): $1-8$.

14 Yoon BH, Romero R, Kim CJ, Jun JK, Gomez $\mathrm{R}, \mathrm{Choi} \mathrm{JH}$, et al. Amniotic fluid interleukin-6: a sensitive test for antenatal diagnosis of acute inflammatory lesions of preterm placenta and prediction of perinatal morbidity. Am J Obstet Gynecol. 1995 Mar;172(3):960-70.
15 Hitti J, Tarczy-Hornoch P, Murphy J, Hillier SL, Aura J, Eschenbach DA. Amniotic fluid infection, cytokines, and adverse outcome among infants at 34 weeks' gestation or less. Obstet Gynecol. 2001 Dec;98(6):1080-8.

16 Romero R, Yoon BH, Kenney JS, Gomez R, Allison AC, Sehgal PB. Amniotic fluid interleukin-6 determinations are of diagnostic and prognostic value in preterm labor. Am J Reprod Immunol. 1993 Sep-Oct;30(2-3):16783.

17 Han YW, Shen T, Chung P, Buhimschi IA Buhimschi CS. Uncultivated bacteria as etiologic agents of intra-amniotic inflammation leading to preterm birth. J Clin Microbiol. 2009 Jan;47(1):38-47.

18 Gravett MG, Witkin SS, Haluska GJ, Edwards JL, Cook MJ, Novy MJ. An experimental model for intraamniotic infection and preterm labor in rhesus monkeys. Am J Obstet Gynecol. 1994 Dec;171(6):1660-7.

19 Figueroa R, Garry D, Elimian A, Patel K, Sehgal PB, Tejani N. Evaluation of amniotic fluid cytokines in preterm labor and intact membranes. J Matern Fetal Neonatal Med. 2005 Oct;18(4):241-7. 